\title{
Are Classes of Nodes with Different Power Amplifiers Good for Wireless Multi-hop Networks?^
}

\author{
Martin Kubisch, Holger Karl, and Adam Wolisz \\ Telecommunication Networks Group \\ Technische Universität Berlin \\ Sekr. FT 5-2, Einsteinufer 25 \\ 10587 Berlin, Germany \\ \{kubisch, karl, wolisz\}@ee.tu-berlin.de
}

\begin{abstract}
It has been shown that optimization of wireless network operations can be achieved by transmission power control of wireless nodes. Controlling the transmission power is mostly performed as a regulation of the final power amplifier, which is the major power sink. Depending on the desired transmission power level, currently popular amplifiers work at different levels of efficiency: highest in case of maximum emitted power, lower in case of reduced emitted power.

In this paper we present a novel approach for improving the liftime of mobile devices in multi-hop wireless networks by using nodes with heterogeneous efficiency characteristics of their power amplifiers.

To assess the potential of such amplifier-heterogeneous networks, we have performed multi-hop network simulations under the idealistic assumption of optimal routing based on global knowledge. The obtained results demonstrate an appealing potential of a reduction in energy consumption to less than $40 \%$ compared to classical networks with uniform amplifiers.
\end{abstract}

Keywords: Multi-hop wireless networks, power control, sensor network.

\section{Introduction}

Today's popular power amplifiers used in wireless network cards are designed to have the highest power efficiency at the maximum output power. When the output power is reduced, the power efficiency of the amplifier decreases, i.e., the power consumed by an amplifier does not reduce with the same ratio as the output power decreases. As an example: the RF2155 power amplifier [1], which is designed for cordless phones and other application in the $915 \mathrm{MHz}$ ISM band, has four different output power levels. The power efficiency of these power level range from $54 \%$ efficiency for the highest level of output power to $1 \%$ efficiency for the lowest level of output power.

Power amplifiers are usually chosen according to the maximum range one wants to overcome. Assuming a desired PER and other parameters of the receiver characteristic, this leads to a requirement on the output power necessary to overcome the maximal

\footnotetext{
* This work is funded by the German Ministry of Education and Research (BMBF) under the project IBMS ${ }^{2}$.
} 
distance. In realistic setups however, the distances between communicating nodes are variable. Thus, the usage of the highest output power is rarely necessary, even when direct communication between the sender and receiver is possible. Further options for using lower transmission power appear when (as in IEEE 802.11) the coding/modulation might be dynamically adjusted (slower data rates require lower transmission power to achieve the same PER). In addition, the usage of multi-hop schemes instead of direct communication offers even more freedom for using lower output power. It is well known that reducing the output power is beneficial because of both reduction of the energy of the transmitter as well as reduction of the potential interference with other nodes operating in the same frequency band.

The desired and beneficial reduction of the output power does, unfortunately enough, not lead to a proportional reduction of power used to drive the amplifier, as the amplifier is moving into a less efficient operation range. But, in fact, there is no physical rule that mandates that power amplifiers have the highest efficiency at the highest output power. A practical example for a different amplifier designs is CRIPPS [2], which presents amplifiers which are developed with efficiency enhancement techniques in mind. The presented "Doherty" amplifier is an example of an adaptation of power efficiency. It has the highest power efficiency at a power level $6 \mathrm{~dB}$ less than the maximum one. Obviously, using such amplifiers with shifted power efficiency would pay off if — mostly — lower output power is applied, even if un-proportionally high power consumption for the highest power level would be used.

As this could be extended with the use of dynamically adjustable characteristics of power amplifiers to result in an optimal selection for a specific network topology, we constrain ourselves only to nodes with two types of amplifiers, further referred to as short- or long-range specialists (note that even the short-range specialists in our model can transmit at the highest power if necessary, but the costs are comparably high). Intuitively, such a mixture of nodes might improve energy efficiency. In this paper, we want to assess whether this intuition actually holds: we study a varying percentage of long- and short-range specialist nodes at various node densities and study the resulting energy efficiency in a wireless network.

Our considerations are structured as follows: first we give a short overview of related work proving that a differentiation of output power level is an attractive way to optimize wireless networks. Further we describe the methodology of this study, details of our simulation set up and some encouraging results. As this paper pertains to work in progress, we complete our considerations with comments on ongoing investigations.

\section{Related Work}

There exist numerous possibilities for reduction of the communication energy in multihop wireless networks. We will not discuss the papers which put their nodes into sleep, even if this is an interesting functionality of some MAC designs (including IEEE 802.11); it can be combined with the approach discussed in this paper.

Relevant to this paper are approaches pertaining to the selection of the transmission power level. This might be globally unified for the whole network, e.g. with respect to desired connectivity [3] [4] or individually for each pair of nodes [5] [6] [7] [8] [9]. [10] 
As shown in [5] and [3], the use of lower power level, which shorten the distance and require more intermediate hops, can increase the energy efficiency as well as the capacity of a network. Especially the consideration of the remaining battery capacity can lead to an extension in network lifetime. Another approach is the COMPOW protocol [4] which exchanges life messages on separate, discrete power levels to find a common power level throughout the network. Unlike these networks based on IEEE 802.11, is the search for the minimum power level of a node [6] a more general approach, which can be applied to low data rate networks as well, e.g., sensor networks. They determine the power level while relying on measurements of the received signal power strength. Additionally to this power level calculation, [7] uses a path recalculation in every node to determine if it could provide a more energy-efficient path.

A proven way of finding a minimum transmission power is done in [9] and [10]. While these algorithms rely either on location information or on angle of arrival, the algorithms in [8] do not. There, locally available information is used to adapt the power level in an sensor network and it is shown that this increases the network lifetime.

Out of this partial list one can see that setting different transmission power levels is beneficial for the energy consumption and other optimization criteria. When optimizing the power setting it is important to understand that mostly the emitted power (assuring a certain SNR at the receiver, defined by a desired PER) is used as the optimization criterion.

In all of these papers it has been assumed that the parameters of the amplifier of all nodes are identical, and only individual settings are possible. To our knowledge, this is the first paper considering a mixture of nodes with different types of amplifier characteristics.

\section{Evaluation Based on Global Knowledge}

The system scenario under consideration is a wireless multi-hop network, e.g., a mobile ad hoc network. Nodes want to communicate with other nodes over longer distances than is possible even with the highest transmission power, necessitating multi-hop communication. For such networks we need an ad hoc routing protocol which chooses energyefficient routes and thus the nodes can use transmission power level small enough to safely communicate with their next hop neighbor.

The advantage of such energy-efficient routing schemes is that the reduction of distance, useful for multi-hop communication, implies a reduction of necessary transmission power, but as the efficiency of the final amplifier does decrease as well, the gain is not as high as it could be. Our hypothesis is that this shortcoming can be overcome by using different nodes in the network, equipped with different power amplifiers, optimized for different distances. We claim that such "specialists" for long- and short-range communication will increase the energy efficiency.

Ideally, an ad hoc routing protocol should be modified to exploit the different characteristics of such nodes, routing short-range communication over nodes with amplifiers that operate efficiently at low transmission powers and bridging long distances with other nodes. Developing such an extension to an ad hoc protocol is actually a non-trivial 
activity. Hence, to first obtain an understanding of the principle feasibility and benefits of this approach, we used a simplified approach and examine it.

In our approach we have a fixed number of uniform randomly distributed nodes, where each node has only two output power levels (the output power levels are the same for short- and long-range specialists), and we change the percentage of nodes which are short-range specialists. For each node and every power level in such a network the maximum transmission range is calculated. Using the global knowledge of all node's location, we determine which node can be reached and annotate the edges between the nodes with the energy consumed per packet as the cost function.

Then we use Dijkstra's algorithm to calculate the optimal path between any two nodes in the network, resulting in a forwarding table for every node which also contains the necessary transmission power level. Having these paths, we used the following traffic pattern to determine the total energy used: Every node randomly selects one destination node in the network and transfers one packet to it. At the end, the total consumed energy of all nodes serves as a figure of merit for different network densities and ratio of long-/ short-range specialists.

\section{Model Assumptions}

We have considered various model assumptions here. In all scenarios, 100 nodes are uniform randomly distributed in an area between $5 \mathrm{~km} * 5 \mathrm{~km}$ and $18.25 \mathrm{~km} * 18.25 \mathrm{~km}$. This is equal to network densities between 4 (all nodes can communicate directly) and 0.3 nodes per $\mathrm{km}^{2}$ (lower values result in partitioned networks). The efficiency model for the transmission power is based on values for the RF 2155 power amplifier: Each node has two transmission power levels of $70 \mathrm{~mW}$ and $447 \mathrm{~mW}$. The long-range specialists have an efficiency of $54 \%$ (consumed power of $826 \mathrm{~mW}$ ) for the high power level and $20 \%$ (consumed power of $337.5 \mathrm{~mW}$ ) for the low power level. The short-range specialists have an efficiency of $20 \%$ (consumed power of $2235 \mathrm{~mW}$ ) for the high power level and $54 \%$ (consumed power of $129.6 \mathrm{~mW}$ ) for the low power level. The other values of $-85 \mathrm{dBm}$ receiver sensitivity (implying a PER of $1 \%$ ), $200 \mathrm{~mW}$ reception power and $200 \mathrm{~mW}$ computation power while transmitting are based on the "SieMo S50037 Bluetooth Module" [11]. The amplifier characteristic used in a particular node in a particular scenario depends on the percentage necessary for this scenario, e.g., out of this 100 nodes 10 are selected to be short-range specialists the others are long-range. For the traffic we used a packet size of 1500 byte as well as a immediate acknowledgement of 30 bytes at a data rate of $1 \mathrm{Mbit} / \mathrm{s}$ - taking into account the acknowledgment as well is important because of the heterogeneous energy costs of different devices!

\section{Simulation Results}

Figure 1(a) displays the total energy consumed, each averaged over 40 different random placements of nodes. A path loss coefficient of 3 was used. On the x-axis, the percentage of short-range specialists is displayed and on the y-axis the energy necessary for the used traffic pattern which results in a transfer of 100 packets. The lower line is the energy 


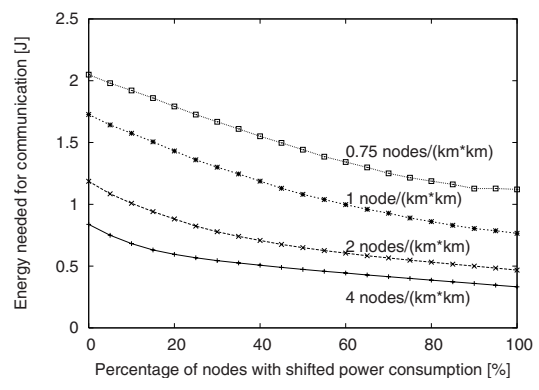

(a) High density

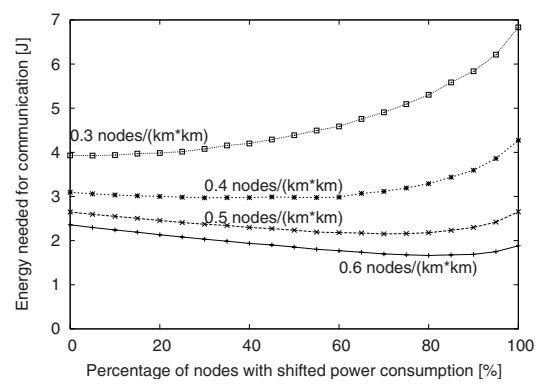

(b) Low density

Fig. 1. Total consumed energy over percentage of short range specialists

average for a density of 4 nodes per $\mathrm{km}^{2}$, the upper one for 0.75 nodes per $\mathrm{km}^{2}$. The lines between are intermediate densities.

For the high-density networks, using only short-range specialists is beneficial and the energy needed is less then $38.4 \%$ compared to a network having only long-range ones.

Figure 1(b) also displays the total energy consumed over the percentage of shortrange specialists, but the networks are less dense. The lower line is the energy average for a density of 0.6 nodes per $\mathrm{km}^{2}$, the upper one for 0.3 nodes per $\mathrm{km}^{2}$.

The three lower lines are the energy curves where the network density is sparse and using only short-range specialists is not most beneficial. Instead, there is an optimal point depending on the density. For a density of 0.6 nodes per $\mathrm{km}^{2}$ this ratio is $80 \%$, for 0.5 nodes per $\mathrm{km}^{2}$ the ratio is around $70 \%$ and for 0.4 nodes per $\mathrm{km}^{2}$ it is around $30 \%$. When the density is 0.3 nodes per $\mathrm{km}^{2}$ or smaller, it is not beneficial to use short-range specialists at all. Additionally, further reduction in density leads to another problem: the connectivity. When a much lower density is used, the probability of having a disconnected network becomes higher, thus further curves are left out.

\section{Conclusions and Further Work}

As the results show, heterogeneity of nodes with differently optimized power amplifiers are beneficial for energy efficiency. The best ratio between long- and short-range specialists depends on the density of the network, the node layout (one could imagine a network where particular nodes are of better use when they adapt their amplifier characteristic) and also, implicitly, on the characteristics of the different amplifiers. Thus, it is a complex problem, but we intend to tackle it step by step.

Although this idea was demonstrated for WLAN networks, we believe it could also be applied to other wireless networks, e.g., sensor networks. In these networks the problem is quite similar apart from the fact that other packet sizes and traffic patterns are used.

As a next step we intend to integrate the characteristics of such different short- and long-range specialists into ad hoc routing protocols. The particular challenge is to handle the different energy costs for both directions in a simple and efficient manner. Moreover, 
the application of such a heterogeneous nodes approach should be particularly useful in wireless sensor networks, where the density of the network can be estimated beforehand.

In such a network, also the use of cluster-based routing protocols is very popular, and it might be attractive to use the long-range specialists as clusterheads.

Acknowledgement. We thank Prof. G. Böck and W. Chen for their discussion regarding power amplifiers.

\section{References}

1. RF Micro Devices: RF 2155 3V Programmable Gain Power Amplifier. Designer's Handbook (2003) 2-173-2-180

2. Cripps, S.C.: RF Power Amplifiers for Wireless Communications - Efficiency Enhancement Techniques. Artech House Publishers, Boston, MA, USA (2000)

3. Monks, J.P., Ebert, J.P., Wolisz, A., Hwu, W.: A study of the energy saving and capacity improvement potential of power control in multi-hop wireless networks. In: Workshop on Wireless Local Networks, Tampa, Florida, USA, also Conf. of Local Computer Networks (LCN). (2001)

4. Narayanaswamy, S., Kawadia, V., Sreenivas, R.S., Kumar, P.R.: Power control in ad-hoc networks: Theory, architecture, algorithm and implementation of the COMPOW protocol. In: European Wireless Conference, Florence, Italy, EUREL, VDE (2002) 156-162

5. Kubisch, M., Mengesha, S., Hollos, D., Karl, H., Wolisz, A.: Applying ad-hoc relaying to improve capacity, energy efficiency, and immission in infrastructure-based WLANs. In Irmscher, K., ed.: Kommunikation in Verteilten Systemen (KiVS), 13. ITG/GI-Fachtagung, Leipzig, Germany, Informatik Aktuell, Springer (2003)

6. Bergamo, P., Maniezzo, D., Giovanardi, A., Mazzini, G., Zorzi, M.: Distributed power control for power-aware energy-efficient routing in ad hoc networks. In: European Wireless Conference, Florence, Italy, EUREL, VDE (2002) 237-243

7. Doshi, S., Bhandare, S., Brown, T.X.: An on-demand minimum energy routing protocol for a wireless ad hoc network. Mobile Computing and Communications Review 6 (2002) 50-66

8. Kubisch, M., Karl, H., Wolisz, A., Zhong, L.C., Rabaey, J.: Distributed algorithms for transmission power control in wireless sensor networks. In: Wireless Communications and Networking Conference (WCNC), New Orleans, LA, IEEE (2003)

9. Rodoplu, V., Meng, T.H.Y.: Minimum energy mobile wireless networks. IEEE Journal on Selected Areas in Communications 17 (1999) 1333-1344

10. Wattenhofer, R., Li, L., Bahl, P., Wang, Y.M.: Distributed topology control for power efficient operation in multihop wireless ad hoc networks. In: INFOCOM - The Conference on Computer Communications, Anchorage, Ak, IEEE (2001)

11. Siemens AG Austria: Siemens S50037 Bluetooth Module (2001) 